\title{
sciendo
}

\section{IDENTIFICATION OF mRNA DEGRADOME VARIATION DEPENDENT ON DIVERGENT MUSCLE MASS IN DIFFERENT PIG BREEDS*}

\author{
Katarzyna L. Piórkowska ${ }^{1 \star}$, Tomasz Szmatoła ${ }^{1,2}$, Klaudia Pawlina-Tyszko ${ }^{1}$, Artur Gurgul ${ }^{1,2}$, \\ Grzegorz Żak ${ }^{3}$, Katarzyna M. Ropka-Molik ${ }^{1}$ \\ ${ }^{1}$ Department of Animal Molecular Biology, National Research Institute of Animal Production, \\ 32-083 Balice n. Kraków, Poland \\ ${ }^{2}$ University Centre of Veterinary Medicine, University of Agriculture in Krakow, \\ Al. Mickiewicza 24/28, 30-059 Kraków, Poland \\ ${ }^{3}$ Department of Pig Breeding, National Research Institute of Animal Production, \\ 32-083 Balice n. Kraków, Poland \\ •Corresponding author: katarzyna.piorkowska@izoo.krakow.pl
}

\begin{abstract}
The search is still on for the molecular processes associated with the development and metabolism of skeletal muscles. Selection conducted in farm animals is focused on high muscle mass because it delivers higher economic profit. The present study aimed to shed light on mRNA degradome signals that could be characteristic for molecular processes associated with an abundance of muscle mass and to identify miRNA regulatory networks controlling these processes in pigs applying next-generation-sequencing (NGS). In the study, over 10,000 degraded transcripts were identified per sample, with the highest abundance for genes encoding mitochondrial proteins (COXs, NDs, CYTB, ATP6 and ATP8). Moreover, only $26 \%$ of the miRNA targets were found within this degraded transcript pool, which suggested for miRNAs other molecular mechanism at different level of gene expression than mRNA degradation. On the other hand, a small share of the identified degraded transcripts associated with miRNA regulation suggests a different mechanism of mRNA degradation for identified degraded transcropts. Subsequently, most of the miRNA gene degraded targets, such as $E N O 3, C K M, C R Y A B$ and $A D A M 19$ encode proteins involved in the muscle mass control. The present study showed an interesting dependence between miRNAs and their targets. Nevertheless, the complete view of the miRNA regulatory network could be a subject of further advanced research, which would employ a miRNA transfection procedure in skeletal muscle cell cultures.
\end{abstract}

Key words: degradome, miRNA, skeletal muscle, muscle fibre

*The study was supported by the statutory activity of National Research Institute of Animal Production (01-18-03-21). 
Muscle mass in pigs has been the most improved production trait over recent decades. Animals with increased muscle mass are much more valuable because they bring more economic profit. In Poland, pigs used as maternal components, such as Polish Large White (PLW) and Polish Landrace (PL), achieve approximately 60\% meat content in the carcass. In turn, sire line pig populations selected particularly for meat content have reached 63\% (POLSUS - Polish Pig Breeders and Producers Association, Report 2018). However, selection focused on increased meat content is still on, since one of the paternal lines still used in the Polish breeding (Pietrain) reveals a high frequency of T allele regarding $R Y R 1 \mathrm{C}>\mathrm{T}$ polymorphism (Škrlep et al., 2010). Therefore, the development of a new sire line isolated from available active populations with high muscle mass potential seems to be essential. Thus, the search for molecular processes associated with the determination of skeletal muscle (SM) development in pigs and the identification of potential genetic markers related to high muscle mass can significantly support this action.

Over recent years, next-generation sequencing methods have been used to identify proteins/genes engaged in molecular processes associated with SM development. Numerous mutations, candidate genes, non-coding transcripts and miRNAs related to myogenesis were identified (Palstra et al., 2013; Qian et al., 2015; Ropka-Molik et al., 2018; Zhi et al., 2005). miRNAs are particularly interesting molecules for investigation due to not fully explored capabilities. Initially, it was believed that miRNAs regulate only mRNA degradation. However, recent studies reported that miRNA has many more functions than mRNA degradation (Eulalio et al., 2009; Freimer et al., 2018). mRNA degradation is a fundamental process in living cells that is associated with maturation, mRNA quality control and gene expression regulation. The pool of digested mRNA in the cell is classified as the mRNA degradome (Jackowiak et al., 2011). The cellular transcript level is defined as a resultant of RNA synthesis and its degradation. The complexity of transcripts regulation involving RNA degradation and the role of miRNA in this process remains not exactly clear (Gebert and MacRae, 2019). On the other hand, Roy and Jacobson (2013) suggest that the vast number of molecular processes leading to mRNA degradation have unknown regulatory mechanisms. The miRNA molecules are loaded into RNA-induced silencing complexes (RISCs), which link to transcripts through complementary base pairing (Pratt and MacRae, 2009). However, mRNA decay directed by a highly complementary miRNA is a rather plant-specific phenomenon. Nevertheless, a piece of evidence for miRNA-directed mRNA cleavage was also found in mammals, since human miR196 binds to $H O X B 8$ leading to its degradation (He et al., 2011). Recently, the muscle mRNA degradome was analysed in Sus scrofa in the context of miRNA regulation. However, the authors focused on the identification of miRNA-target genes (Ye et al., 2018).

The present study used next-generation sequencing (NGS) technology to identify the mRNA degradome signals that could be characteristic for molecular processes associated with muscle mass control in pigs. The present investigation focused on miRNA regulation and identification of the other degraded transcripts under a different unknown kind of control. 


\section{Material and methods}

\section{Animal and sample collection for degradome sequencing}

The present study included 12 pigs belonging to three breeds differing in muscularity: Pietrain $(n=4)$, Hampshire $(n=4)$ and Polish Large White (PLW, $n=4)$. In each breed, half individuals were characterized by low muscle mass (LMM) and a half in high muscle mass (HMM). Pigs belonged to one gender (female) and were maintained at the Pig Test Station under the same feeding and housing conditions up to $100 \pm 2 \mathrm{~kg}$ of body weight. The loin samples were collected up to $20 \mathrm{~min}$ and stabilized in RNAlater solution (Ambion, Cambridge, UK) and frozen at $-20^{\circ} \mathrm{C}$. Total RNA and miRNA were isolated for transcriptome and miRNAome analyses as described in the previous study by Ropka-Molik et al. (2018). Loin mass was assessed according to Tyra and Żak (2013). The experimental pipeline is presented in Figure 1.

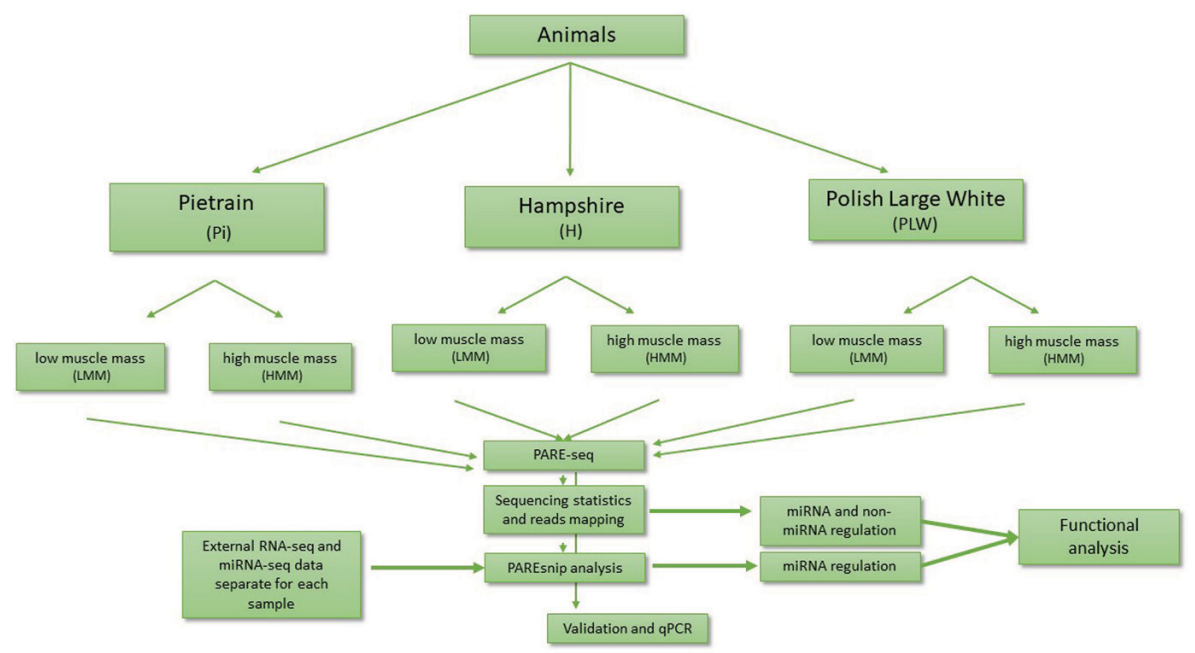

Figure 1. Experimental pipeline for detecting the degraded transcripts under miRNA and non-miRNA regulations

\section{RNA isolation and parallel analysis of RNA ends (PARE) library construc- tion}

PARE sequencing, described by Zhai et al. (2014) delivers information on $20 \mathrm{bp}$ fragments of degraded transcripts that begin with nuclease cut sites under miRNA and non-miRNA control. PARE library preparation is possible due to the isolation of polyA tail-ended molecules and the ligation of a $5^{\prime}$ RNA adapter only to degraded transcripts with an uncapped $5^{\prime}$ end. The analysis requires a large amount of RNA (40,000-75,000 ng). Therefore, a few RNA isolations were performed for each animal in the present study. We decided to use $65 \mu \mathrm{g}$ of total RNA as the input. RNA was isolated with TRI Reagent Solution (Ambion, Cambridge, UK), and using beads homogenization and 1-bromo-3-chloropropane (Sigma-Aldrich, Saint Louis, USA) 
instead of chloroform. The isolated RNA was suspended in DEPC-treated water. The RNA integrity number (RIN) values estimated by TapeStation2200 and RNA tapes (Agilent, Palo Alto, USA) were in the range of 7.5-8.5. The PARE libraries were prepared as described by Zhai et al. (2014) with some modifications. TargetRT, $5{ }^{\prime}$ and $3^{`}$ DNA PCR, FinalPCR and PAREseq primers and the 5 'RNA adapter were HPLC purified and purchased from Genomed (Poland). dsDNA_bottom 5 -CCT TGG CAC CCG AGA ATT CCA NN-3' and dsDNA_top 5 '-TGG AAT TCT CGG GTG CCA AGG-3` primers were PAGE purified (Invitrogen). In addition, we used ready-to-use $10 \%$ Novex $^{\mathrm{TM}} \mathrm{TBE}$ Gels for both electrophoreses that were run on the XCell SureLock ${ }^{\circledR}$ Mini-Cell (Invitrogen, Carlsbad, USA) in 0.5\% TBE. PARE library quantification was assessed by TapeStation2200 (D1000 tape) (Agilent, Palo Alto, USA) and by Qubit ${ }^{\mathrm{TM}}$ using a dsDNA HS Assay (Invitrogen, Carlsbad, USA). The final concentration of the PARE libraries was normalized to $10 \mathrm{nM}$. The libraries were then pooled, diluted to $10 \mathrm{pM}$ and loaded into a v3 Illumina flow cell in two lanes (two technical replicates) on a cBot cluster station (Illumina). Flow cell clustering was performed using a TruSeq SR Cluster Kit v3-cBot-4 HS. Sequencing-bysynthesis of the clustered libraries was conducted on a HiScanSQ System in a single 80-bp run using TruSeq SBS Kit v3-HS chemistry (Illumina Inc., San Diego USA) accompanied by the $25 \%$ of Phix control library (Illumina Inc., San Diego, USA).

\section{Aligning raw reads to the pig genome}

The quality control of raw reads was performed using FastQC software, and then they were mapped to Sus scrofa genome (version 11.1) assembly together with a GTF annotation (v94) using TopHat 2 software (Trapnell et al., 2009) set for singleend reads. The quality of RNA-seq mappings was controlled using RSeQC software (Wang et al., 2012). We used only reads that mapped to the pig genome for further analysis with the PAREsnip program (part of the UEA small RNA Workbench) (Stocks et al., 2018). This analysis required information on miRNAome and transcriptome for each sample generated in a previous study (Ropka-Molik et al., 2018) (GSE109215). PAREsnip identified miRNA cut sites based on the Sus scrofa 11.1 genome assembly, and the annotation of genes was performed using Biomart in Ensembl browser.

\section{miRNAome and transcriptome analyses}

The sequencing of the whole miRNA and mRNA molecules in Pietrain and Hampshire pigs was performed as described previously by Ropka-Molik et al. (2018) (GSE107207, GSE109215). The same analysis, which included miRNA and transcriptome sequencing was performed for Polish Large White pigs according to the procedure described by Ropka-Molik et al. (2018) using eight gilts showing high $(\mathrm{n}=4)$ and low $(\mathrm{n}=4)$ loin mass (unpublished).

The pig groups significantly differed in the relationship to muscle mass content. For example, high muscle mass (HMM) Pietrain pigs were characterised by approximately $2 \mathrm{~kg}$ heavier ham compared to low muscle mass (LMM) group. In turn, LMM Hampshire gilts had $8 \mathrm{~cm}^{2}$ smaller loin eye areas, and the difference in meat content within PLW pigs was 9\% (Table 1). 
Table 1. The meat traits of examined pigs

\begin{tabular}{l|c|c|c|c|c|c}
\hline \multicolumn{1}{c}{ Breed/trait } & $\begin{array}{c}\text { PLW } \\
\text { LMM }\end{array}$ & $\begin{array}{c}\text { PLW } \\
\text { HMM }\end{array}$ & $\begin{array}{c}\text { Pietrain } \\
\text { LMM }\end{array}$ & $\begin{array}{c}\text { Pietrain } \\
\text { HMM }\end{array}$ & $\begin{array}{c}\text { Hampshire } \\
\text { LMM }\end{array}$ & $\begin{array}{c}\text { Hampshire } \\
\text { HMM }\end{array}$ \\
\hline Loin mass & $5.08 \pm 0.28 \mathrm{a}$ & $6.18 \pm 0.57 \mathrm{~b}$ & $5.32 \pm 0.12 \mathrm{a}$ & $6.05 \pm 0.12 \mathrm{~b}$ & $4.96 \pm 0.04 \mathrm{~A}$ & $7.07 \pm 0.11 \mathrm{~B}$ \\
Ham mass & $8.30 \pm 0.26 \mathrm{~A}$ & $10.1 \pm 0.07 \mathrm{~B}$ & $8.65 \pm 0.05 \mathrm{~A}$ & $10.37 \pm 0.01 \mathrm{~B}$ & $8.85 \pm 0.47 \mathrm{a}$ & $10.54 \pm 0.35 \mathrm{~b}$ \\
Loin eye area & $49.3 \pm 4.05$ & $58.0 \pm 8.15$ & $63.8 \pm 0.35$ & $64.8 \pm 0.65$ & $55.4 \pm 4.20$ & $63.4 \pm 3.55$ \\
Meat percentage & $58.9 \pm 2.15 \mathrm{a}$ & $68.0 \pm 2.35 \mathrm{~b}$ & $64 \pm 1.65 \mathrm{~A}$ & $72.4 \pm 0.001 \mathrm{~B}$ & $63.4 \pm 2.05$ & $66.2 \pm 0.60$ \\
\hline
\end{tabular}

Loin and ham muscle weights are given in kilograms. Loin eye are is given in $\mathrm{cm}^{2}$. HMM - high muscle mass, LMM - low muscle mass. A, B - P-value $<0.01$, a, b - P-value $<0.05$. The used animals were selected from numerous population in order to present extreme values describing meat content.

For mRNA molecules, we used only reads that mapped to the pig genome for further analysis with the PAREsnip program.

\section{Identification of miRNA-degraded transcripts}

A Venn diagram (Bioinformatics \& Evolutionary Genomics, Belgium) was used to pinpoint the shared miRNAs between analysed groups. The information on the whole pool and differentially expressed miRNA molecules related to muscle mass development was described by Ropka-Molik et al. (2018) and is available under the link goo.gl/W6uLxk. Specific miRNA-targets that could be correlated with muscle mass development were predicted for each analysed group. The functional analysis of the identified miRNA targets and other degraded mRNAs was performed using STRING (functional protein association networks) (Szklarczyk et al., 2019) and Panther Classification System (Mi et al., 2019). In both tools default settings were used with False Discovery Rate (FDR) $<0.05$ and $S$. scrofa reference.

\section{The validation of identified miRNA-degraded transcripts}

The validation was performed using cDNA that was synthesized during PARE library preparation. It was amplified twice using $5^{\prime} \mathrm{cDNA}$ PCR -GTT CAG AGT TCT ACA GTC CGA C and 3 'cDNA CGA GCA CAG AAT TAA TAC GAC T PCR primers and Phusion Hot Start II polymerase (Zhai et al., 2014) to yield nothing except degraded transcripts flanked by adaptors. Sanger sequencing was performed on gene fragments amplified by gene-specific primers using ABI 3500XL genetic analyser (Applied Biosystems, Foster City, USA) and BigDye Terminator v3.1 Cycle Sequencing Kit (Applied Biosystems, Foster City, USA). The primer sequences are shown in Table S1 (https://bit.ly/3lk3Ba5). The bam files were generated by SAMtools software (Li et al., 2009) and visualised by Integrative Genomics Viewer (IGV v. 2.3).

\section{Results}

\section{Sequencing statistics and reads mapping}

The average $94.4 \%$ total number of raw reads/sample were mapped to the pig reference genome (Sus scrofa 11.1). There were identified over 10,000 degraded 
transcripts per sample, with the highest abundance for genes encoding mitochondrial proteins involved in cell respiration, such as COXs, NDs, CYTB, ATP6 and ATP8, and also non-mtDNA proteins involved in development of skeletal muscle such as ACTA1, MYH2, MYL1 and PYGM (goo.gl/W6uLxk). Moreover, non-mtDNA genes that showed high share within degraded mRNA pool (top 30) in HMM pigs were associated with muscle filament contraction - GO:0006936 (MYOM2, NEB, DES, TTN, TNNI2, MYH4, TNNT3 and ALDOA), glycolysis/gluconeogenesis (ENO3, $A L D O A, P G M 1, G O T 2, P F K M$ and $G P I)$ and muscle chronological ageing (CA3, $P G M 1, E N O 3, M Y O M 2, T N N T 3$ and TTN) (Figure 2). In turn, negatively correlated with muscle mass mRNA decay were found for $M Y B P C$ and $C K M$ transcripts that also related to muscle mass development. The clustering of samples and estimated degraded transcript abundance was presented by heatmaps (Figure 3). The experiment was submitted to GEO NCBI database (GSE129681).

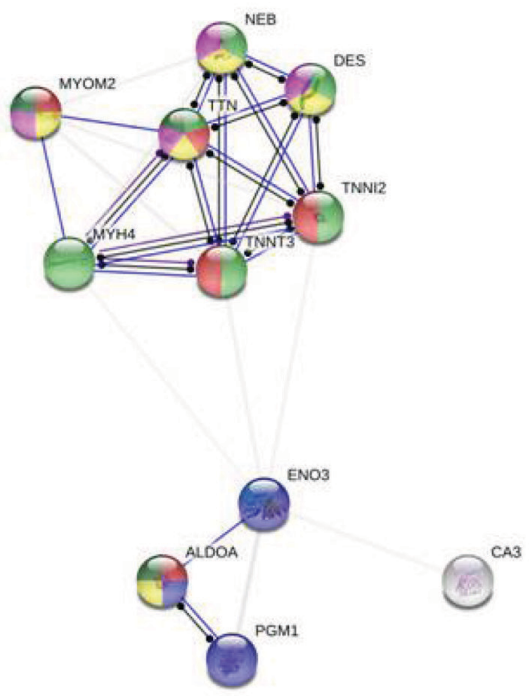

GO:0031430 - $\mathrm{M}$ band

GO:0006096 -glycolytic process

G0:0030049 -muscle filament sliding

GO:0031674 -I band

GO:0006941 -striated muscle contraction

GO:0030018-Z disc

Figure 2. Network of interaction between the most interesting non-mtDNA proteins encoded by transcripts that showed the highest degree of degradation in high muscle mass (HMM) pig groups. The colours indicate the involvement of particular proteins encoded by degraded transcripts in important for muscle mass development biological process and pathways. The network was presented as a scalable vector graphic and based on Homo sapiens reference 


\section{PARE sequencing statistics}

The PAREsnip analysis revealed 713 degraded transcript fragments on average corresponding to 168 genes (Table S2, https://bit.ly/3lk3Ba5). The comparison of the miRNAome(Ropka-Molik etal., 2018) with PAREsnip results showed that only $26 \%$ of miRNA targets within the degraded gene pool were identified (goo.gl/W6uLxk). In the high muscle mass PLW and Hampshire pigs, much fewer miRNA targets were identified than in low muscle mass group, and the opposite result was found in Pietrain pigs (Table S3, https://bit.ly/3lk3Ba5).
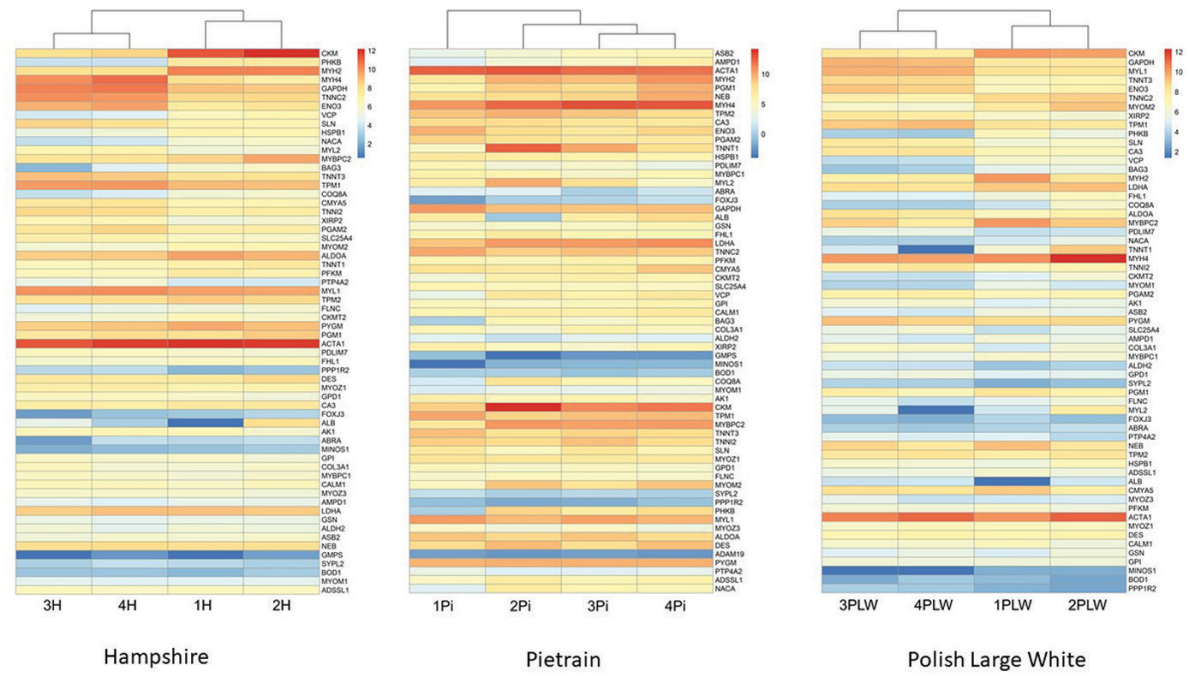

Figure 3. Heatmap showing sample clustering based on normalized reads for Hampshire Pietrain and Polish Large White pigs. The degradation pattern obtained for the most significant (involved in muscle mass development) transcript detected in Hampshire breed in pigs groups differed in muscle mass ( 1 and 2 - low muscle mass, 3 and 4 - high muscle mass). (R Package pheatmap, R Foundation for Statistical Computing, Vienna, Austria). The reads were normalized by input number reads

\section{Identification of miRNA target genes for all detected miRNA}

The PAREsnip analysis indicated a few shared miRNAs for which targets were identified (Figure 4). In LMM Hampshire and PLW, degradation of ADAM19, MYOM2 and GMPS transcripts corresponding to miR-23a regulation was identified. In turn, degraded $C R Y A B$ and $H S P B 2$ transcripts were re-lated to miR-125a activity in both HMM Pietrain and LMM PLW pigs. The shared miRNAs for which target genes were identified, are shown in Figure 4 and Table S4 (https://bit.ly/3lk3Ba5). 


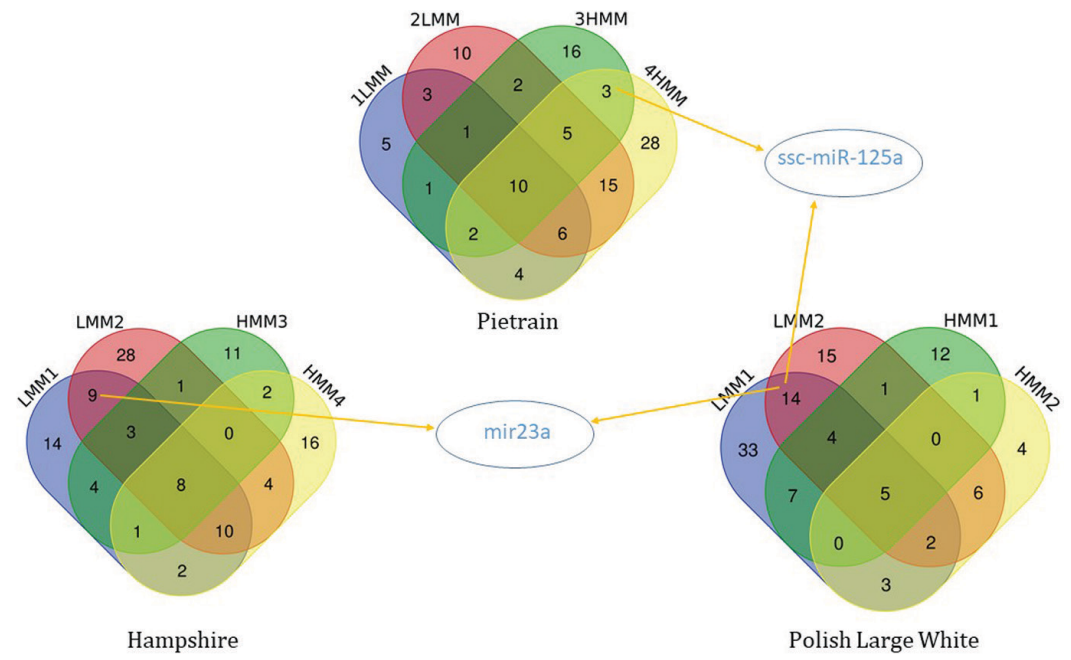

Figure 4. Venn diagram presenting number of miRNAs that regulate mRNA degradation. In ellipses were shown shared miRNAs for two different pig groups that presented various muscle mass

\section{Identification of miRNA target genes for DE miRNAs}

In the previous study, Ropka-Molik et al. (2018) identified numerous miRNAs that were differentially expressed (DE) in response to variable muscle mass in pigs. In the Pietrain pigs, it was found that ssc-miR-30a-5p correlated with higher muscle mass leading to SYPL2 (MG29) transcript decay, which was identified in the present study. The protein encoded by SYLP2 gene is involved in communication between the T-tubular and junctional sarcoplasmic reticulum (SR). In the Hampshire pigs, it negatively correlated with muscle mass, the ssc-miR-127 likely controlled the degradation of $F O X J 3$ transcript. Moreover, numerous targets for positively correlated with muscle mass content ssc-miR-23b were identified - ADAM19 in LMM pigs and AIMP1 in HMM pigs. Furthermore, ENO3, BOD1 and ND5 transcript degradation were identified that could correspond to miR-769-5p, miR-493-5p and let-7c miRNA, respectively. The enolase 3 (ENO3), biorientation of chromosomes in cell division 1 (BOD1) and NADH dehydrogenase 5 (ND5) proteins are involved in muscle mass control and regeneration (Figure 5).

\section{Identification of miRNA target transcripts corresponding to more than one miRNA}

The PAREsnip analysis identified signals from cleaved TPM1, CA3 and PPP1R2 transcripts in each of the investigated samples (goo.gl/W6uLxk) corresponding to different miRNA molecules in particular group. Degraded MYLK2 and C12orf45 transcripts were found in both LMM PLW and LMM Hampshire pigs that were associated with ssc-miR-4331, NA24/mmu-miR-1983, NA25/miR-1983. In turn, the cleavage of COL $3 A 1$ transcript was observed in HMM Pietrain and LMM PLW pigs. In addition, the signal of degradation of $M Y H 2$ transcript was identified in HMM Pietrain pigs, and Myh2 is a protein that is related to skeletal muscle contraction process. 


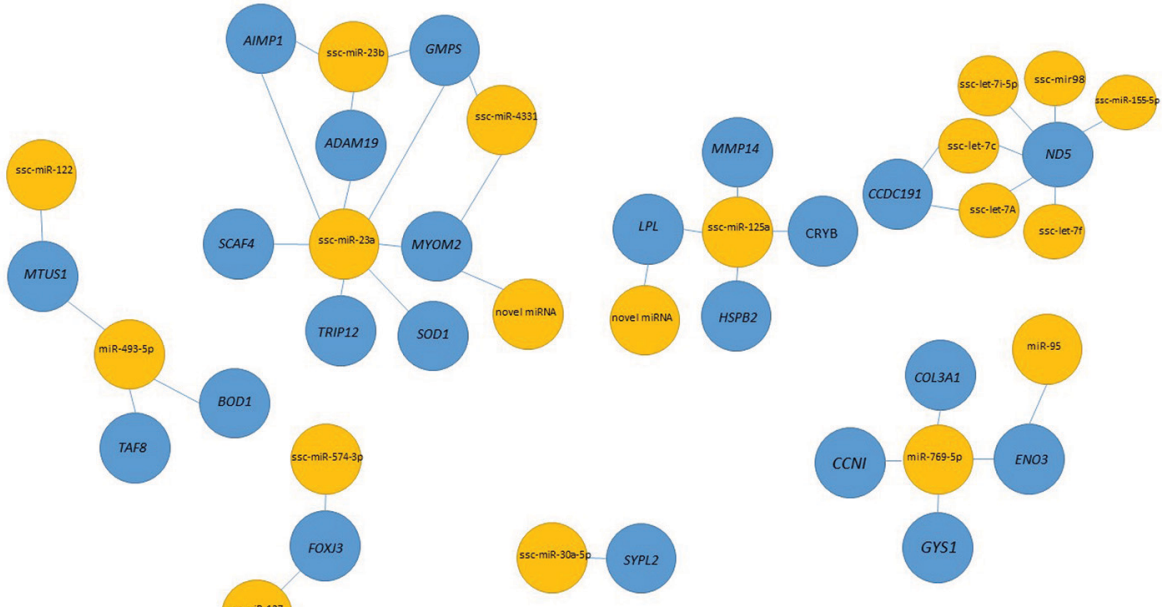

Figure 5. Network of miRNAs and their target identified in degradome sequencing and predicted based on PAREsnip analysis

\section{Validation}

The presence of five degraded transcripts was tested using Sanger sequencing. Figure 5 presents the bam files visualised by IGV2 v.2.3 after PARE/degradome sequencing analysis and chromatograms of the PCR products generated using nested PCR. Sanger sequencing confirmed the presence of ABRA, CA3, ENO3, TPM1 and $P P P 1 R 2$ transcripts in their degraded forms (Figure 6). The genes/transcripts were randomly chosen for the validation, and they showed both low and high degrees of decay.
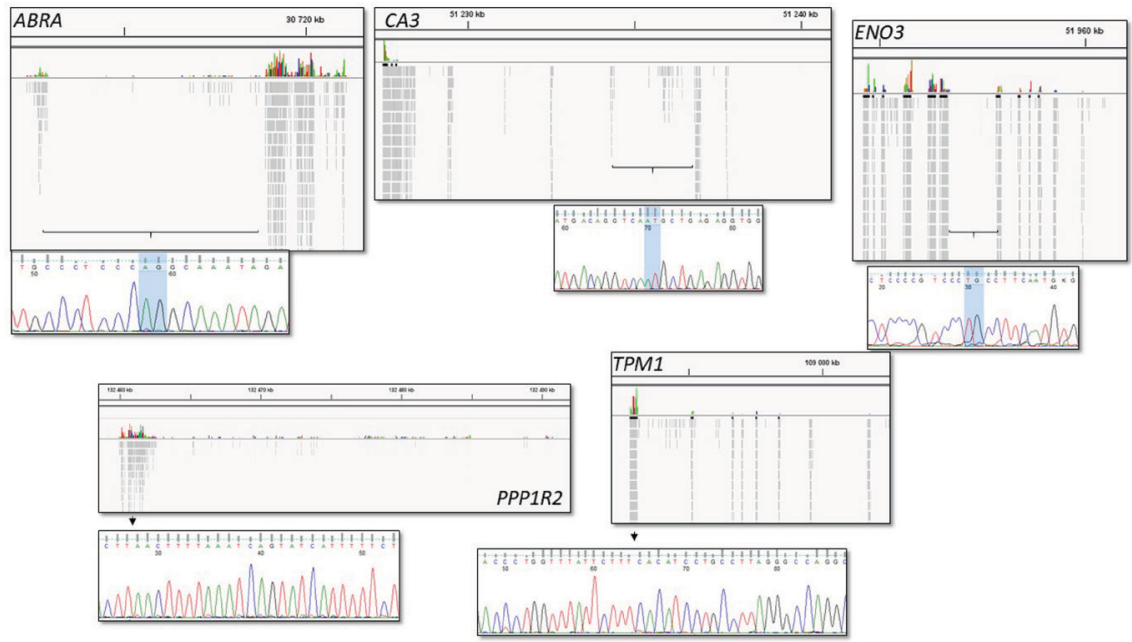

Figure 6. Visualized by IGV2 v. 2.3 bam files showing degraded transcripts obtained after degradome sequencing analysis and fragments of Sanger sequencing chromatograms, which confirmed the presence of degraded transcripts. The blue shadows indicated the exon junctions. In the PPPIRI and TPMI primers were designed for the 3'UTR regions 


\section{Discussion}

A miRNA is an interesting molecule that regulates gene expression. In plants, these small RNAs bind precisely to the complementary 3' UTR of the messenger RNA, which results in direct cleavage. In animals, miRNAs bind to mRNAs in an imprecise complementary manner (Ambros, 2004). In the beginning, it was believed that miRNA inhibits gene expression only throughout transcript degradation. However, Olsen and Ambros (1999) found that miRNA molecules lead to translational repression by blocking the release of mRNAs from ribosomes. Previously, it was considered also that animal miRNAs bind to target transcripts mainly in the 3'UTR region, but insightful studies provided evidence that they can also target coding sequences or 5'UTRs (Lytle et al., 2007). Recently, Sanei and Chen (2015) indicated reciprocal regulation between miRNAs and their target genes, which can influence miRNA decay. In turn, Ameres and Zamore (2013) supported that the degree of complementarity between the miRNA and its target mRNA appears to be crucial for transcript degradation, and in the animal kingdom, a miRNA induces translational repression rather than gene silencing. Over recent years, the conducted studies provided evidence that gene silencing mechanisms, RNA decay and translation inhibition, occur in both the plant and animal kingdoms. Based on this assumption, bioinformatic/genetic consortiums offer various tools for the prediction of miRNA regulation using the information on degraded mRNAs generated by NGS methods, such as PAREsnip (part of the UEA small RNA Workbench) (Stocks et al., 2018) and Cleave Land (Addo-Quaye et al., 2009).

The present study attempted to identify the degradome signal associated with divergent muscle abundance by using high and low muscle content pigs. Although the conducted research was only preliminary in this field, interesting results were obtained. The sequencing of PARE libraries identified increased mRNA decay level for ENO3, TNNT3 and TTN and lowered for MYH2, MYBPC2 and CKM transcripts in HMM pigs. It was interesting because these genes are strongly related to abundance of skeletal muscle mass. TTN encodes titin, a protein involved in muscle contraction. It is the giant sarcomeric protein anchoring the $\mathrm{Z}$ line to the $\mathrm{M}$ line and is responsible for the passive elasticity of the muscle (Krüger and Kötter, 2016). Titin is correlated with different kinds of myopathies and dystrophies in humans (Savarese et al., 2016). In turn, van der Pijl et al. (2018) described the functions of titin as a mechanosensor that regulates muscle trophicity because it interacts with LIM and ankyrin repeat proteins (Krüger and Kötter, 2016). In addition, Wu et al. (2018) postulated that the white muscle is fit for short and strong contractile performance due to high levels of titin, whereas the red muscle is fit for low intensity and long-lasting activity due to high levels of lipids and mitochondria. Thus, different degrees of TTN decay can be associated with different fibre diameter in heavier muscles (Wu et al., 2018). In turn, the present PAREsnip analysis indicated the possible regulation of TTN expression by ssc-miR-935, but it was observed only in Pietrain pig with increased muscle mass. Moreover, the troponin 3 (TNNT3) fast skeletal type is associated with initialization of the muscle contraction process that is dependent on $\mathrm{Ca}^{2+}$ ions changing the conformation of troponin 3 (Gurnett et al., 2009). However, we did not find any 
miRNA regulation for TNNT3 in the present study. In turn, ENO3, which encodes enolase 3 , is believed to play a role in muscle development and regeneration. In humans, its mutations are correlated with glycogen storage diseases (Adeva-Andany et al., 2016). The present PAREsnip study demonstrated that ENO3 mRNA decay is probably controlled by miR-769-5p or miR-95 (Figure 5). Interestingly, higher degradation of ENO3 was observed in pigs with increased muscle mass. In turn, $C K M$, which encodes creatine kinase, was found to be more degraded in pigs with decreased muscle mass. CKM is a cytosolic protein that also plays a role in energy homeostasis in skeletal muscles. Moreover, a small fraction of CKM is co-localized at the M-band of the sarcomeres neighbouring ENO3 (Foucault et al., 1999). This observation could be associated with the fact that the percentage of muscle fibres and their diameter are dependent on muscle mass and area, for example, in loin (Bereta et al., 2014). However, CKM is expressed in all types of myofibres (Tai et al., 2011) and ENO3 observed in type I fibres.

The present study also attempted to pinpoint the mRNA degradation controlled by miRNAs using PARE sequencing (PARE-seq) and the PAREsnip tool. The preliminary findings of the PAREsnip analysis indicated that only a small percentage of the identified degraded transcripts were probably regulated by miRNAs, which indicates cleavage control by different kinds of small interfering RNAs (siRNAs) (Wilson and Doudna, 2013) or unknown miRNAs that were not detected after miRNA sequencing or a completely different unknown mechanism. Moreover, the miRNA targets were, on average, identified for $26 \%$ of the detected miRNA molecules, which evidences that miRNAs are employed at various molecular levels and are responsible for the induction of translational repression rather than mRNA decay in animals, which was supported by the report of Ameres and Zamore (2013).

A few interesting observations of possible miRNA directed transcript degradation were made in the present study. Two miRNAs, namely miR-23a and miR-125a were found to regulate mRNA degradation in more than one investigated pig breed. Although miR-125a was previously described as affecting glucose uptake in adipose tissue (Diawara et al., 2014) rather than regulating muscle development, it was found to be correlated with augmented muscle mass in Hampshire and PLW pigs, and reduced muscularity in Pietrain gilts in the present study. It was interesting that the identification of miR-125a targets (CRYAB and HSPB2) was correlated with reduced expression of this miRNA molecule. This observation suggests that the increased expression of miR-125a is related to miRNA activity other than mRNA degradation. On the other hand, HSPB2 encodes heat shock protein family B (small) member 2, which regulates myotonic dystrophy protein kinase (MKBP), composing the MKBP/HSPB2 complex and plays a role in the maintenance of myofibril integrity (Sugiyama et al., 2000). In turn, the CRYAB gene also encodes small heat shock protein that interacts with the $\mathrm{N}$ and $\mathrm{C}$ termini of Ago2, serves as a part of the RISC complex and is necessary for cellular homeostasis in skeletal muscle (Neppl et al., 2014). In addition, a shift in the hypertrophy-atrophy signalling axis towards atrophy was observed in the skeletal muscle of CryAB-null mice (Neppl et al., 2014). The obtained findings indicate a distinct manner of gene expression regulation associated with muscle development in Pietrain pigs compared to other investigated breeds. The 
comparison of muscle fibre microstructure between Pietrain and Polish Large White pigs indicated significant differences in fibre diameters of all types, but not in fibre composition, which is associated with hypertrophic phenomenon (Orzechowska et al., 2008), although Te Pas et al. (2005) observed a longer muscle fibre proliferation stage in foetuses of Pietrain pigs, which was in turn characteristic for hyperplasia (Coutinho et al., 1993). The second miRNA, miR-23a, was not found to be differentially expressed, but it was identified in muscles of the PLW and Hampshire pigs with decreased muscle mass. The PAREsnip analysis indicated that miR-23a is likely associated with the degradation of $A D A M 19$ and MYOM2 transcripts. Interestingly, ADAM19 encodes the ADAM metallopeptidase domain 19, which is implicated in various biological processes, including inter alia muscle development. Neuner et al. (2009) observed that $A D A M 19$ knock-down may interfere with muscle differentiation in Xenopus, which was related to a low level of myosin light chain (MLC) expression. Whilst, myomesin 2 (MYOM2) is an M-line part as an accompanying titin protein in fast fibres (Fukuzawa et al., 2008). In turn, Liu et al. (2016) found that its expression was enhanced during muscle development. In the present study, additional miRNA regulatory molecules of ADAM19 and MYOM2 were identified (miR-23b and miR-4331, respectively). The comparison of miRNA target genes between the low and high muscle mass pigs showed numerous transcripts characteristic for specific group. $M Y L K 2$, which was found as degraded in both Hampshire and Polish Large White pigs with reduced muscle mass under miR-4331 regulation can also play a role in skeletal muscle mass development. It encodes $\mathrm{Ca}^{2+} /$ calmodulindependent myosin light chain kinase 2, which is involved in the phosphorylation of myosin light chain, a primary biochemical mechanism for tension potentiation due to repetitive stimulation in fast-twitch skeletal muscles (Zhi et al., 2005).

The complex analysis comparing all miRNA targets showed that the non-mitochondrial TPM1, CA3 and PPP1R2 degraded transcripts were identified in each sample. $C A 3$ encodes carbonic anhydrase that was probably regulated by miR-378, which was described previously as negatively correlated with muscle mass because the overexpression of miR-378 leads to a reduction in the size and number of muscle fibres. In turn, muscle regeneration was attenuated by cardiotoxin, which was associated with delayed satellite cell activation and differentiation (Zeng et al., 2016). The present PAREsnip analysis showed that TPM1 mRNA decay was under miR-376 control, whose expression was modified by endurance training exercises.

Pigs are a popular animal model because they better reflect the physiological changes occurring in the human body in comparison to small laboratory animals, due to their similar body proportions (Groth, 2007). Additionally, pigs were successfully used as a model in muscular dystrophy (Selsby et al., 2015) and atrophy (Duque et al., 2015), therefore the present study could be valuable in researches solving the issue of human skeletal muscle disorders.

\section{Conclusion}

The present study showed an interesting dependence between miRNAs and their targets. Numerous transcripts that were degraded are involved in the increasing skeletal muscle mass and degradation of ENO3, MYLK, ADAM19 and TPM1 can be key 
in the regulation during controlling this process. Moreover, the present study confirmed that most miRNAs in pigs are associated with other kinds of gene expression control rather than transcript degradation. We also showed another kind of transcript cleavage regulation, because the PAREsnip analysis showed that only a moderate percentage of the identified degraded transcripts were associated with miRNA regulation. However, the complete view of the miRNA regulation network could be obtained in a more advanced study that employs miRNA transfection procedures in muscle cell cultures, which could be a subject of further research.

\section{Conflict of interests}

The authors declare no conflicts of interest.

\section{Declarations}

\section{Ethics approval and consent to participate}

The research was performed on biological material derived from pigs maintained and slaughtered in the Test Pig Station (National Research Institute of Animal Production). In the Test Station pigs are slaughtered, dissected and after carcass evaluation, meat is standard intended for consumption. Therefore, our research does not require the approval of the Animal Experimentation committee.

\section{Consent for publication}

Not applicable

\section{Availability of data and material}

Data available in GEO database GSE107207(https://www.ncbi.nlm.nih.gov/geo/ query/acc.cgi?acc=GSE107207), GSE109215 (https://www.ncbi.nlm.nih.gov/ geo/ query/acc.cgi), GSE129681 and under link goo.gl/W6uLxk. For the others, please contact authors.

\section{References}

A d d o-Qu a y e C., M ille r W., A x te 11 M.J. (2009). CleaveLand: a pipeline for using degradome data to find cleaved small RNA targets. Bioinformatics, 25: 130-131.

Ad e va - A nd a n y M.M., G o n zál e z - L u cá n M., Donapetry-García C., Fernández-Fernández C., Ameneiros-Rodríguez E. (2016). Glycogen metabolism in humans. BBA Clin., 5: $85-100$.

A m bros V. (2004). The functions of animal microRNAs. Nature, 431: 350-355.

A mere s S.L., Zamore P.D. (2013). Diversifying microRNA sequence and function. Nat. Rev. Mol. Cell Biol., 14: 475-488.

Bereta A., Tyra M., Ropka-Molik K., Wojtysiak D., Różycki M., Eckert R. (2014). Histological profile of the longissimus dorsi muscle in Polish Large White and Polish Landrace pigs and its effect on loin parameters and intramuscular fat content. Ann. Anim. Sci., 14: 955-966.

C o ut in ho L.L., M or r is J., Mark s H.L., B u hr R.J., I vari e R. (1993). Delayed somite formation in a quail line exhibiting myofiber hyperplasia is accompanied by delayed expression of myogenic regulatory factors and myosin heavy chain. Development, 117: 563-569. 
Di aw ara M.R., Hue C., Wilder S.P., Venteclef N., A ron-Wisnewsky J., S cott J., Clé m e n t K., G a u g u i er D., C a ld e r a r i S. (2014). Adaptive expression of microRNA-125a in adipose tissue in response to obesity in mice and men. PLoS One, 9.

Duque S.I., Arnold W.D., Odermatt P., Li X., Porensky P.N., Schmelzer L., Meyer K., Kolb S.J., Schümperli D., Kaspar B.K., Burghes A.H.M. (2015). A large animal model of spinal muscular atrophy and correction of phenotype. Ann. Neurol., 77: 399-414.

Eulali o A., Huntzinger E., N ishihara T., Rehwinkel J., F a user M., I zaurralde E. (2009). Deadenylation is a widespread effect of miRNA regulation. RNA, 15: 21-32.

Foucault G., Vacher M., Merkulova T., Keller A., Arrio-Dupont M. (1999). Presence of enolase in the M-band of skeletal muscle and possible indirect interaction with the cytosolic muscle isoform of creatine kinase. Biochem. J., 338 ( Pt 1): 115-121.

Fre imer J.W., H u T.J., B lelloch R. (2018). Decoupling the impact of MicroRNAs on translational repression versus RNA degradation in embryonic stem cells. Elife, 7: e38014.

Fukuzawa A., Lange S., Holt M., Vihola A., Carmignac V., FerreiroA., Udd B., Gautel M. (2008). Interactions with titin and myomesin target obscurin and obscurin-like 1 to the M-band - Implications for hereditary myopathies. J. Cell Sci., 121: 1841-1851.

G e b e r t L.F.R., M a c R a e I.J. (2019). Regulation of microRNA function in animals. Nat. Rev. Mol. Cell Biol., 20: 21-37.

Groth C.G. (2007). The potential advantages of transplanting organs from pig to man: A transplant surgeon's view. Indian J. Urolog., 23: 305-309.

Gurnet t C.A., A l a e e F., D e sru is se a u D., B o e hm S., D obbs M.B. (2009). Skeletal muscle contractile gene (TNNT3, MYH3, TPM2) mutations not found in vertical talus or clubfoot. Clin. Orthop. Relat. Res., 467: 1195-1200.

He X., Yan Y.L., Eberhart J.K., Herpin A., Wagner T.U., Schartl M., Postlethw a it J.H. (2011). MiR-196 regulates axial patterning and pectoral appendage initiation. Dev. Biol., 357: 463-477.

J a c kow iak P., N ow a ck a M., S trozy ck i P.M., F ig lerow ic z M. (2011). RNA degradomeits biogenesis and functions. Nucleic Acids Res., 39: 7361-7370.

K r ü g e r M., K ö t t e r S. (2016). Titin, a central mediator for hypertrophic signaling, exercise-induced mechanosignaling and skeletal muscle remodeling. Front. Physiol., 7: 76.

L i H., H and sake r B., Wy s o ke r A., F e nne 11 T., R u a n J., Ho m er N., M a r th G., A b e c a s i s G., D u r b in R. (2009). The Sequence Alignment/Map format and SAMtools. Bioinformatics, 25: 2078-2079.

Li u J., F u R., Li u R., Z h a o G., Zh eng M., C u i H., Li Q., S ong J., Wang J., Wen J. (2016). Protein profiles for muscle development and intramuscular fat accumulation at different post-hatching ages in chickens. PLoS One, 11.

Ly t 1 e J.R., Y Y r i o T.A., S te i t z J.A. (2007). Target mRNAs are repressed as efficiently by microRNA-binding sites in the 5' UTR as in the 3' UTR. Proc. Natl. Acad. Sci. USA, 104: 9667-9672.

Mi H., Muruganuj an A., Eb er t D., Hu ang X., Thom a s P.D. (2019). PANTHER version 14: More genomes, a new PANTHER GO-slim and improvements in enrichment analysis tools. Nucleic Acids Res., 47: D419-D426.

N e p p 1 R.L., K a t a o k M., Wa n g D.Z. (2014). Crystallin- $\alpha$ B regulates skeletal muscle homeostasis via modulation of Argonaute2 activity. J. Biol. Chem., 289: 17240-17248.

Neuner R., Cousin H., McCusker C., Coyne M., Alfandari D. (2009). Xenopus ADAM19 is involved in neural, neural crest and muscle development. Mech. Dev., 126: 240-255.

Ols e n P.H., A m bro s V. (1999). The lin-4 regulatory RNA controls developmental timing in Caenorhabditis elegans by blocking LIN-14 protein synthesis after the initiation of translation. Dev. Biol., 216: 671-680.

Orzechowska B., Wojtysiak D., Migdał W., Tyra M. (2008). Relationships between muscle fibre characteristics and physico-chemical properties of longissimus lumborum muscle and growth rate in pig fatteners of three breeds. Anim. Sci. Pap. Rep., 26: 277-285.

Palstra A.P., B eltran S., B urgerhout E., Brittijn S.A., Magnoni L.J., Henkel C.V., Jansen H.J., van den Thillart G.E.E.J.M., S paink H.P., P lan as J.V. (2013). Deep RNA sequencing of the skeletal muscle transcriptome in swimming fish. PLoS One, 8: e53171. 
Pratt A.J., M a c R a e I.J. (2009). The RNA-induced silencing complex: A versatile gene-silencing machine. J. Biol. Chem., 284: 17897-17901.

Qian L., Tang M., Yang J., Wang Q., Ca i C., Jiang S., Li H., Jiang K., G a o P., Ma D., C h e n Y., A n X., L i K., C u i W. (2015). Targeted mutations in myostatin by zinc-finger nucleases result in double-muscled phenotype in Meishan pigs. Sci. Rep., 5: 14435.

Ropka-Molik K., Pawlina-Tyszko K., Żukowski K., Piórkowska K., Żak G., Gurgul A., Derebecka N., We s oły J. (2018). Examining the genetic background of porcine muscle growth and development based on transcriptome and miRNAome data. Int. J. Mol. Sci., 19: 1208 .

R o y B., J a c obs on A. (2013). The intimate relationships of mRNA decay and translation. Trends Genet., 29: 691-699.

S a n e i M., Ch e n X. (2015). Mechanisms of microRNA turnover. Curr. Opin. Plant Biol., 27: $199-206$.

S a varese M., S arparanta J., Vihola A., Udd B., Hackman P. (2016). Increasing role of titin mutations in neuromuscular disorders. J. Neuromuscul. Dis., 3: 293-308.

S e l s b y J.T., Ros s J.W., N onneman D., Hollinger K. (2015). Porcine models of muscular dystrophy. ILAR J., 56: 116-126.

Š k r le p M., K a va r T., Č a n d e k - P o t o k a r M. (2010). Comparison of PRKAG3 and RYR1 gene effect on carcass traits and meat quality in Slovenian commercial pigs. Czech J. Anim. Sci., 55: $149-159$.

S to c k s M.B., Mohorianu I., B e ckers M., Pa i cu C., Moxon S., Thody J., Dalmay T., M o u l t o n V. (2018). The UEA sRNA Workbench (version 4.4): a comprehensive suite of tools for analyzing miRNAs and sRNAs. Bioinformatics, 34: 3382-3384.

Sugiyama Y., Suzuki A., Kishikawa M., Akutsu R., Hirose T., Waye M.M.Y., Ts u i S.K.W., Y o s hid a S., Ohno S. (2000). Muscle develops a specific form of small heat shock protein complex composed of MKBP/HSPB2 and HSPB3 during myogenic differentiation. J. Biol. Chem., 275: 1095-1104.

Szklarczyk D., Gabl A.L., Lyo D., Junge A., Wyde S., Huerta-Cepas J., Simonovic M., Don cheva N.T., M orris J.H., B ork P., J en se L.J., M er in g C. von (2019). STRING v11: protein-protein association networks with increased coverage, supporting functional discovery in genome-wide experimental datasets. Nucleic Acids Res., 47: D607-D613.

Tai P.W.L., F i s her-Aylor K.I., H i meda C.L., S m i th C.L., M a c K en zi e A.P., Hel t e r line D.L., Angello J.C., Weliks on R.E., Wold B.J., Hauschka S.D. (2011). Differentiation and fiber type-specific activity of a muscle creatine kinase intronic enhancer. Skelet. Muscle, 1: 25 .

Te Pas M.F.W., Cagnazzo M., De Wit A.A.C., Priem J., Pool M., Davoli R. (2005). Muscle transcriptomes of Duroc and Pietrain pig breeds during prenatal formation of skeletal muscle tissue using microarray technology. Arch. Tierz., Dummerstorf., 48: 141-147.

Trapnel1 C., Pachter L., S a lzberg S.L. (2009). TopHat: Discovering splice junctions with RNA-Seq. Bioinformatics, 25: 1105-1111.

Tyra M., Żak G. (2013). Analysis of the possibility of improving the indicators of pork quality through selection with particular consideration of intramuscular fat (IMF) content. Ann. Anim. Sci., 13: 33-44.

van der Pijl R., Strom J., Conijn S., Lindqvist J., Labeit S., Granzier H., Ott e n h e ij m C. (2018). Titin-based mechanosensing modulates muscle hypertrophy. J. Cachexia Sarcopenia Muscle, 9: 947-961.

W a n g L., W a n g S., L i W. (2012). RSeQC: Quality control of RNA-seq experiments. Bioinformatics, 28: $2184-2185$.

Wi 1 s o n R.C., D o u d n a J.A. (2013). Molecular mechanisms of RNA interference. Annu. Rev. Biophys., 42: 217-239.

Wu M.P., Chang N.C., Chung C.L., Chiu W.C., H s u C.C., Chen H.M., She u J.R., J a y a k u m a r T., C h o u D.S., F o n g T.H. (2018). Analysis of titin in red and white muscles: crucial role on muscle contractions using a fish model. Biomed Res. Int., https://doi.org/10.1155/2018/5816875

Ye R.S., Li M., Chen T., Wei X.C., Qi Q.E., Cheng X., Li C.Y., Jiang Q.Y., Xi Q.Y., $\mathrm{Z}$ h a $\mathrm{ng}$ Y.L. (2018). miRNAome, mRNAome and degradome analysis of Tibetan minipigs anterior pituitary. Gen. Comp. Endocrinol., 259: 104-114. 
Zeng P., Han W., Li C., Li H., Zhu D., Zhang Y., Li u X. (2016). MiR-378 attenuates muscle regeneration by delaying satellite cell activation and differentiation in mice. Acta Biochim. Biophys. Sin. (Shanghai), 48: 833-839.

Z ha i J., A rikit S., S i m on S.A., K ing ha m B.F., Me ye r s B.C. (2014). Rapid construction of parallel analysis of RNA end (PARE) libraries for Illumina sequencing. Methods, 67: 84-90.

Z hi G., Ry d e r J.W., H u ang J., D ing P., Ch en Y., Z h a o Y., K a m m K.E., S tu 11 J.T. (2005). Myosin light chain kinase and myosin phosphorylation effect frequency-dependent potentiation of skeletal muscle contraction. Proc. Natl. Acad. Sci. USA, 102: 17519-17524.

Received: 21 I 2020

Accepted: 25 VI 2020 